\title{
Set Theoretical Structures of Physical Concepts
}

\author{
Tetsuo SHIMIzU
}

Fujitsu

\section{Fundamental Structure of Physical World}

Physical world is constructed as collection of physical events. A physical event is decomposed into a product of physical attributes such as epoch, position, brightness, color, temperature, and so on. We introduce a set theoretical view to describe those physical events. Every physical measurement of the event is represented by an element in Cartesian product of sets corresponding to the physical attributes. Therefore we can represent a physical event $e$ as

$$
e=\left(a_{1}, a_{2}, \cdots, a_{n}, \cdots\right) \in A_{1} \times A_{2} \times \cdots \times A_{n} \times \cdots,
$$

where $a_{1}, a_{2}, \cdots, a_{n}, \cdots$ are elements of sets $A_{1}, A_{2}, \cdots A_{n}, \cdots$ corresponding to the physical observables.

In this context we can represent our physical observations into the form of the table as follows :

\begin{tabular}{c|c|c|c|c}
\hline $\begin{array}{c}\text { physical } \\
\text { event }\end{array}$ & $\begin{array}{c}\text { space of physical } \\
\text { attribute } A_{1}\end{array}$ & $\begin{array}{c}\text { space of physical } \\
\text { attribute } A_{2}\end{array}$ & $\cdots$ & $\begin{array}{c}\text { space of physical } \\
\text { attribute } A_{n}\end{array}$ \\
\hline$e$ & $a_{1}$ & $a_{2}$ & & $a_{n}$ \\
$e^{\prime}$ & $a_{1}^{\prime}$ & $a_{2}^{\prime}$ & & $a_{n}^{\prime}$ \\
$\vdots$ & $\vdots$ & $\vdots$ & $\vdots$ \\
$e^{(m)}$ & $a_{1}^{(m)}$ & $a_{2}^{(m)}$ & & $a_{n}^{(m)}$ \\
$\vdots$ & $\vdots$ & $\vdots$ & & $\vdots$ \\
\hline
\end{tabular}

We call the Cartesian product generalized configuration space in which all physical events are represented. Caotic physical world is represented as a free Cartesian product of any possible combinations of the configuration spaces. Physical formulation is to classify the free product into several universal representatives; i.e. relations.

Set theoretical relation is represented as a subset of Cartesian product by

$$
R_{i j} \subset A_{i} \times A_{j}
$$

and, more generally,

$$
R_{i 1, i 2, \cdots i n}, \cdots \subset A_{i 1} \times A_{i 2} \times \cdots \times A_{i n} \cdots .
$$

Physical laws are represented by some set theoretical relations in generalized configuration space. In other words, physics concerns how to restrict the free product of sets of physical attributes into subset with coordinated physical relations, and physical theory deals with geometric and algebraic structure of the related subset in the generalized configuration space. 
Now we conventionally decompose the sets of physical attributes into two parts ; one part consisting of epoch and position and another part consisting of the others. Epoch is an element of time and position is an element of space. Concept of time and space is most fundamental in physics because we can scarsely imagine physical phenomena without these physical attributes.

This fact is extremely emphasized in classical mechanics, where all physical objects are described as world lines in spacetime and all physical quantities are derived from differential operations to the world lines.

On the contrary, epoch and position of events are not privileged physical quantities in case of quantum mechanics. World points as physical observables are related with energy-momentum operators by quantization equation such as $[p, q]=$ $\hbar / i$, where $p$ is energy momentum operator and $q$ is spacetime position operator. Spacetime position of quantum event is measured with uncertainty. This statement presents that not only local (differential) structure of spacetime but also global (integral) structure is important to describe microscopic physical events.

Another part of the generalized configuration space corresponds to frame in which usual physical quantities are represented. We reconstruct spacetime structure at first in section 2 , and the other physical configuration space next in section 3 .

\section{Spacetime Structure}

2.1 Locality of Physical Observation and Concept of Manifold

All physical events occure in finite time interval and spatial region. Our physical experiences are constrained to the finite fraction of spacetime. How can we recognize whole spacetime? We find a key to treate the whole spacetime in the concept of manifold.

Manifold is locally coordinated topological space defined as follows ;

1) $M$ is topological space with topology $T$.

2) $\bigcup_{\alpha} U_{\alpha}=M\left(U_{\alpha} \in T\right)$ and for $U_{\alpha}$ there exists a coordinate mapping $m_{\alpha}: U_{a} \rightarrow \boldsymbol{R}^{n}$. For $U_{\alpha} \cap U_{\beta}, m_{\alpha} m_{\beta}^{-1}$ is a coordinate transformation of $\boldsymbol{R}^{n}$. In other words manifold is a patch-work consisting of local domains.

Manifold structure is an appropriate model of our empirical recognition of spacetime. For example $m y$ domain of physical experiences is denoted by $U$ and your one by $V$. If $U \cap V$ is not empty, we are experiencing identical events on $U$ $\cap V$ although they are described in two mutually different frames of reference. If these two frames of reference are equivalent (i.e. isomorphic under coordinate transformation), our domain of experiences is extended to $U \cup V$. If the process of extension is continued successively, we can reach a maximum reigion of physical 
experiences, which is practically universal to us. We can extend our private physical concept, which is available only in the finite region, to the universal paradigmatic one so long as it is conformal to those of others.

\subsection{Time}

We briefly review some concepts about time and logical relations among them in this subsection.

\section{1) Countability of Time}

Time is countable : i.e. there exists an unit 1 in the set of time $T$ such as $t \in$ $T \rightarrow t+1 \in T$. We can experience time through sequence of physical events ;

$e, e^{\prime}, \cdots, e^{(n)}, \cdots$.

Some simple examples of relations in the sequence are as follows ;

a) invariant sequence : $e^{(n)} \sim e^{(n+1)}$

b) periodic sequence : $e^{(n)} \sim e^{(n+N)}(N:$ period $)$

c) increasing sequence : $e<e^{\prime}$, where $\sim$ means a equivalence relation.

A most explicit empirical unit of time is day which corresponds to the periodic alternation of daytime and nighttime, and the statement $t \in T \rightarrow t+1 \in T$ means that any day has the next day. If the process is continued ad infinitum, $T$ has structure of natural number: $N$.

There is another possibility that time is not infinite. If $T$ has period $m$, time system is represented by $N /\{m\}=\{t \mid t \in N, t+m \sim t\}$; i.e. time system is equivalent to closed circle. It is practically impossible to determine if time system is finite or infinite because we cannot experience the whole next days.

Of course there is one more possibility that the next day does not exist ; i.e. $\exists$ $t \in T \rightarrow t+1 \notin T$. Some cosmologists say that our world will swallen by black holes and time will not be as we are experiencing now.

Therefore we must satisfy ourselves with saying that time is locally isomorphic to $N$ or, equivalently, locally countable.

2) Dividability of time

Time may have another unit $1^{\prime}$ such that $t+1^{\prime} \in T$. Practically time system has many units such as century, year, month, day, and so on. In the case epoch is represented by Cartesian product $(a, b) \in N \times N$ corresponding two units $\left(1,1^{\prime}\right) \in$ $\boldsymbol{N} \times \boldsymbol{N}$. Dividability of time is equivalent to classification of $N \times \boldsymbol{N}$ by relation ( $a$, $b) \sim\left(a^{\prime}, b^{\prime}\right) \longleftrightarrow a b^{\prime}=a^{\prime} b$. The equivalence class has a representative denoted by $a /$ $b$. Time is represented by concept of rational numbers : $P=\boldsymbol{N} \times \boldsymbol{N} / \sim$, if it is dividable.

If time has structure of rational numbers, it has no minimum interval. Practically all physical phenomena are accompanied with finite duration of the observation time because an observation with complete time resolution requires 
infinite amount of energy as predicated by quantum mechanics. But dividability is very convenient concept we need to introduce continuity of time.

3) Continuity of time

Continuity of time is defined as topological extension of dividability. Convergent Cauchy sequence of rational numbers does not always have its limit point in the field of rational numbers because it is neither algebraic nor topologically closed. The concept of rational numbers is not appropriate enough to represent the concept of time in this context.

Continuity of time is realized in the concept of real numbers: $\boldsymbol{R}$, which is derived from that of rational numbers adding all limit points of Cauchy sequences. The procedure of extension is transcendental because power of $\boldsymbol{R}$ is $2 *$ and that of $\boldsymbol{P}$ is $\boldsymbol{X}$. Although continuity of time includes transcendental posit of Dedekind cut, it is necessary to introduce differential structure on which almost all physical theories of nowadays are built.

\section{4) Differentiability of time}

Differentiability of time is very obvious if it is represented by $\boldsymbol{R}$. But it may happen that time is neither uniform at every point nor independent on physical events. In physics it is supposed that time is locally $C^{\infty}$-differentially isomorphic to $\boldsymbol{R}$. Thus we have reached the most generallized concept of time : one dimensional $C^{\infty}$-differential manifold $M^{1}$.

We can depict logical evolution sequence of the concept of time as follows ;

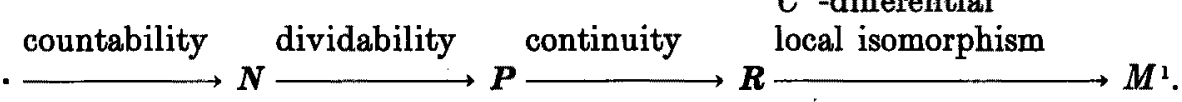

\subsection{Space}

Space is measured by amount of spatial movement although it sounds tautology. The concept of spatial movement is decomposed into distance and direction. Because measurement procedure of distance is always accompanied with duration of time, distance is locally isomorphic to time. Therefore distance is countable, dividable, continuous, and differential because it is spatial representation of time. Thus the physical attribute specific to space is the concept of many degrees of freedom of displacement; i.e. directions.

\section{1) Concept of Path}

Movement is at first realized as a path in space. In other words path is memory of movement and therefore locally isomorphic to $\boldsymbol{R}$.

2) Concept of Euclidian plane

Plane is spanned by deformation of path from its baseline and represented by Cartesian product of path; i.e. $\boldsymbol{R}^{2}$. If minimum distance between two points on the plane is defined by Euclidian measure, it is called Euclidian plane $\boldsymbol{E}^{2}$. 


\section{3) Aristotle's concept of space}

Aristotle's space is represented by $E^{2} \times R$, where $E^{2}$ is Euclid plane corresponding to the degrees of freedom for the horizontal motions and $\boldsymbol{R}$ is vertical distance from the horizontal plane. In this context, space is not uniform upward or downward reflecting the difference between heaven and hell, although horizontal plane permits uniform motion along straight line on it.

Another feature of Aristotle's concept of space is represented by $S^{2} \times R$, where $S^{2}$ is sphere corresponding to the degrees of freedom for steller objects. An eternal trajectory of the steller object is represented by circular motion in $S^{2}$ whereas an motal motion of terrene object is described by vertical line in $\boldsymbol{R}$.

Therefore Aristotle's concept of space has two mutually different representatives $\boldsymbol{E}^{2} \times \boldsymbol{R}$ and $S^{2} \times R$ reflecting the rigid distinction between the mortal terrene objects and the eternal steller ones.

4) Newton's concept of space

In Newton's concept of space, horizontal plane and vertical direction are merged uniformly into three dimensional Euclid space : $E^{3}$. There is no difference between steller events and terrene ones because they are described in the same frame $E^{3}$. $E$ ${ }^{3}$ is one and only one universal frame of reference in which all physical events occur.

But a little reflection reveales that universe may not be globally $E^{3}$, because we cannot reject the other logical possibilities such that universe is neither globally Euclidian nor infinite. Therefore we might at least state that space is locally isomorphic to $\boldsymbol{E}^{3}$ or, equivalently, three dimensional Riemannian manifold $\boldsymbol{M}^{3}$.

Now logical evolution sequence of the concept of space is depicted as follows;

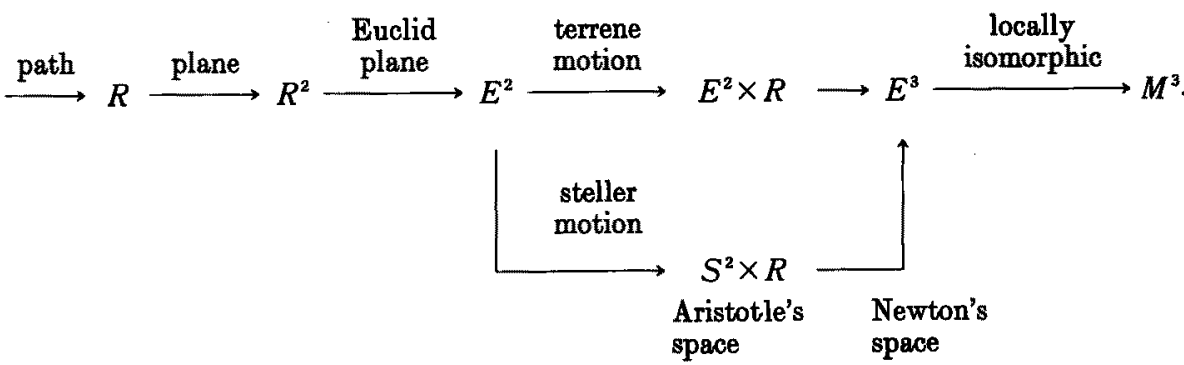

\subsection{Spacetime and Gneralized Concept of Relativity}

Remember that space is measured by movement and the spatial distance is considered as the projection of time. Movement is represented by chronological spatial displacement of identical object as follows: 
observed epoch $: t, t^{\prime}, t^{\prime \prime}, \cdots \in T$

observed position : $x, x^{\prime}, x^{\prime \prime}, \cdots \in S$

observed object : $e, e^{\prime}, e^{\prime \prime}, \cdots\left(e \sim e^{\prime} \sim e^{\prime \prime} \ldots\right.$; identified by an equivalence relar tion $\sim)$.

Worldline is defined as a class of events which can be identified by the equivalence relation. But notify that the identification procedure is possible only in the context of classical mechanics where an observation does not affect status of object. On the contrary the status may be suffered by the successive observations in quantum theory. Therefore strict statement on the concept of spacetime concerns one and only one event described in some mutually different frames of reference.

Any event $e$ is described in local coordinate system of spacetime manifold : i.e. $e(t, x), t \in T, X \in S$. How does another observer describe the event in his frame of reference? If it is denoted by $e^{\prime}\left(t^{\prime}, x^{\prime}\right)$, it must be related with $e(t, x)$ by coordinate transformation $g:(t, x) \rightarrow\left(t^{\prime}, x^{\prime}\right)$ and its representation on the event is defined by $g^{*} e(t, x)=e^{\prime}(g(t, x))$. The last equation shows how the same event is observed by another observer on his frame of reference.

Generalized relativity states that a physical formula denoted by $f$ is invariant under the coordinate transformation : i.e. $f\left(g^{*} e\right)=f(e)$. We will briefly review how the generalized concept of relativity appears in several concepts of spacetime.

1) Aristotle's concept of spacetime

The most primitive style of spacetime is represented by simple Cartesian product of space and time: i.e. $\left(\boldsymbol{E}^{2} \times \boldsymbol{R}\right) \times \boldsymbol{R}$, if mathematical interpretation is possible. Concept of moving frame is not found in his scheme. Physical laws are invariant under horizontal displacement : i.e. transformation $\vec{x}=\vec{x}+\vec{a} \in E^{2}, h^{\prime}=$ $h \in \boldsymbol{R}, t^{\prime}=t \in \boldsymbol{R}$ (time component) preserves quantitas materiae invariant.

2) Galileian concept of spacetime

Concept of moving frame appears at first in Galileian transformation. Galileian relativity is stated as that physical laws are invariant under transformation corresponding to uniform motion on horizontal Euclidian plane as represented by $\vec{x}=\vec{x}-\vec{v} \cdot t \in E^{2}, h^{\prime}=h \in \boldsymbol{R}, t^{\prime}=t \in \boldsymbol{R}$ (time component).

3) Newton's concept of spacetime

Galileian transformation is generalized to be applied to $E^{3}$ in the Newton's concept, where no difference is found to distinguish horizontal and vertical directions. The most simple invariants under the generalized Galileian transformation are relative distance $\overrightarrow{r_{12}}=\left|\overrightarrow{r_{1}}-\overrightarrow{r_{2}}\right|$, time interval $\Delta t=t_{1}-t_{2}$, accelalation $\overrightarrow{a_{12}}=\overrightarrow{a_{1}}-$ $\overrightarrow{a_{2}}$, and Newton's law $\overrightarrow{a_{12}}=F\left(\overrightarrow{r_{12}}\right)$ itself.

4) Einstein's concept of spacetime

Time interval and Eulidian distance are no longer invariant under Lorentz transformation which was historically introduced to describe electromagnetic phoenomena. Lorentz transformation preserves the spacetime distace defined by 
$c^{2}\left(t_{1}-t_{2}\right)^{2}-\left(\overrightarrow{x_{1}}-\overrightarrow{x_{2}}\right)^{2}=\tau_{12}^{2}$, and vector space with the metric is called Minkowski space $M^{4}$. Therefore time $T$ and space $S$ are relative subset of $M^{4}$ : i.e. for $T \subset M$ ${ }^{4}$ and $S \subset M^{4}, g T \neq T$ and $g S \neq S$ where $g$ is Lorentz transformation. This means that time and space are mutually relative concepts depending on the frame of reference of the observer.

\section{5) Spacetime concept in the theory of general relativity}

We reach the final stage of spacetime concept in that of the general relativity, where spacetime structure is characterized by pointwisely defined metric $g^{\mu \nu}(x) d x_{\mu}$ $d x_{\nu}$ for $x \in M . \quad M$ is curved spacetime which is locally isomorphic to Minkowski space.

Because every physical observation is performed on the locally tangent hyperplane denoted by $T x$ for $x \in M$, frame of reference is specific to the spacetime position of the observer. Therefore we must translate the frame of reference through all over $M$ to obtain global structure of $M$. Set of all frames of reference is called tangent bundle $T(M)=\bigcup_{x \in M} T x$, on which all physical events are described. Concept of relativity is redefined as invariance of physical relations under Affine connection of the tangent bundle.

Now logical evolution sequence of the concept of spacetime is shown as follows;

\begin{tabular}{|c|c|c|c|c|c|}
\hline & $\begin{array}{l}\text { Aristotle's } \\
\text { spacetime }\end{array}$ & & $\begin{array}{l}\text { Galileian } \\
\text { spacetime }\end{array}$ & & $\begin{array}{l}\text { Newtons's } \\
\text { spacetime }\end{array}$ \\
\hline $\begin{array}{l}\text { invariance } \\
\text { under } \\
\text { horizontal } \\
\text { translation }\end{array}$ & $\left(E^{2} \times R\right) \times R$ & $\begin{array}{l}\text { invariance } \\
\text { under } \\
\text { uniform } \\
\text { motion }\end{array}$ & $\left(E^{2} \times R\right) \times I$ & $\begin{array}{l}\text { invariance } \\
\text { under } \\
\text { 3-dimensional } \\
\text { uniform } \\
\text { motion }\end{array}$ & $E^{2} \times R$ \\
\hline
\end{tabular}

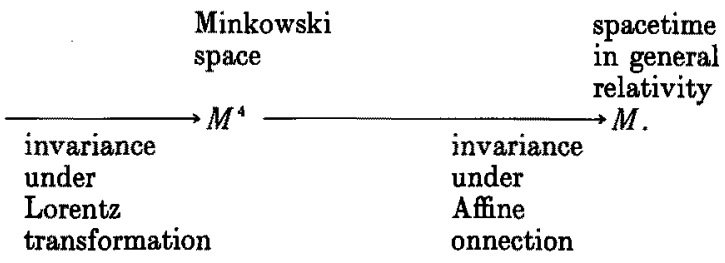

\section{Generalized Configuration Space}

\subsection{Quantitas Materiae and Bundle Structure}

We have decomposed generalized configuration space into two parts ; frame of spacetime and that of quentitas materiae as follows: 


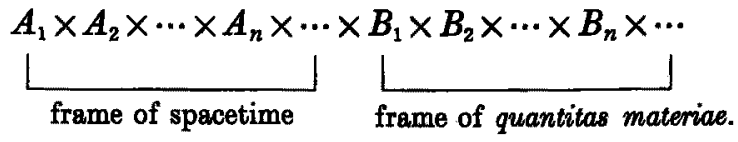

One part concerning the frame of spacetime is analized in the preceding section, and we have finally obtained the concept of spacetime manifold denoted by $M$. We will discuss on the other part of generalized configuration space concerning frame of quantitas materiae.

Because all physical events are always accompanied with finite spacetime region and physicl quantities, we can describe these events in the frame $U_{\alpha} \times F_{\alpha}$, where $U_{a} \in M$ and $F_{\alpha}$ is a frame of physical quantities assigned to $U_{\alpha} . F_{\alpha}$ is called fibre space over $U_{\alpha}$, and the total configuration space $E=\bigcup_{U a \in M} U_{\alpha} \times F_{\alpha}$ is called bundle structure over spacetime $M$. If $F$ exists such that $F \sim F_{a}$ for all $U_{\alpha} \subset M, F$ is called universal fibre over $M$. $F$ presents a paradigmatic structure of generalized configuration space. Therefore a study on generalized configuration space reduces to that of the bundle structure and the fibre space.

In the context of bundle structure, all physical statuses are represented by cross sections of the bundle structure denoted by $\Gamma(E)$, which is subset of $E$ and consists of all elements isomorphic to spacetime; i.e.

$$
\Gamma(E)=\{\gamma \mid \gamma \subset E \& \gamma \stackrel{1: 1}{\longleftrightarrow} M\} .
$$

A cross section is a fibre valued function. It is parameterized by spacetime and represents the configuration of physical object as depicted in the figure as follows:

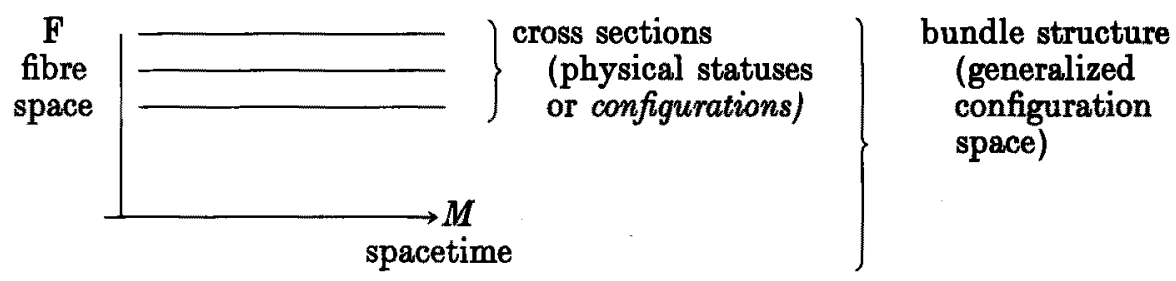

\subsection{Empirical Examples of Bundle Structure}

We will present some examples of applications of bundle structure to some empirical modeles.

\section{1) 2-valued configuration space}

The most simple example of bundle structure is given by $M \times 2$, where 2 is space of two valued logic: i.e. Boolian algebra $(0,1)$. Physical interpretation of fibre space $(0,1)$ is (nonexistence, existence) respectively. $\Gamma(M \times 2)$ is set of the Boolean valued (discontinuous in general) functions over spacetime. Therefore $y \in$ $\Gamma(M \times 2)$ has a representative function $f$ such that;

$$
f(x)=1(x \in U \subset M)
$$




$$
=0(x \in M-U) \text {. }
$$

$f$ is interpreted as that a physical object exists at some spacetime region $U \subset M$. For example, the statement that unicorn exists as an physical object means that state function of unicorn has value 1 at some spacetime region $U \subset M$.

Notice that universal existence (or nonexistence) is equal to 1 -valued (or, equivalently, 0 -valued) constant function of spacetime (respectively). Therefore physical universality means spacetime itself as Newton's god was spacetime itself in his concept.

\section{2) Color-valued configuration space}

Next we present color-valued configuration space as an interesting example of application of bundle structure to empirical model of world recognition. Color space is represented by Cartesian product $C \times B$, where $C$ and $B$ are spaces of color and brightness respectively depicted as follows;

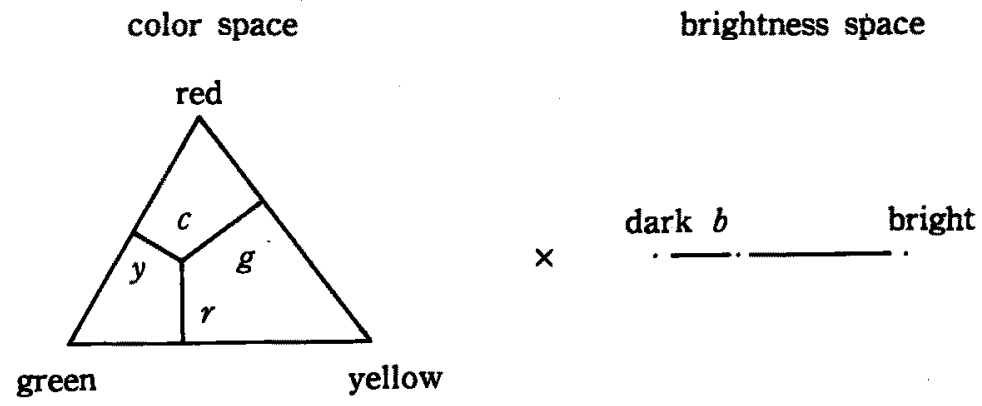

Therefore configuration space is given by $M \times(C \times B)$, where $M$ is spacetime. The statement that there is an object with color $c$ and brightness $b$ at world point $x$ is represented by $(x ; c, b) \in M \times(C \times B)$, where $c$ is decomposed into $(r, g, y)$. Metaphorically speaking, sense data correspond to points in the configuration space. Our world picture is represented by $\gamma \in \Gamma(M \times(C \times B))$ : i.e. cross section of the empirical bundle structure or, equivalently, color-and-brightness valued function of spacetime.

\subsection{Bundle Structures in Physics}

Some examples of bundle structures which appear in physics are as follows.

\section{1) N-valued configuration space}

The most primitive physical quantity we encounter is number of the physical objects which are not mutually distinguishable as individuals. For example, the statement that 'two apples on the table' is invariant if we exchange the spatial positions they occupy. Of course 'two apples on the table' and 'two electrons in the 
vaccuum tube' are not physically equivalent statements because the former is interpreted in classical sense and the latter is in quantum logic.

In classical concept of numbers, there exists a method to distinguish two identical objectives. All classical objects are fundamentally distinguishable because detailed observation would reveal that two objectives are really different. For example we can draw a sign or number on the surface of the apple and the apple stays to be an apple. On the contrary in the quantum concept, we cannot always distinguish two physical objects because distinguishability means that successive observations to identify the object do not alter the status and it is fundamentally impossible.

Bundle structure of $N$-valued configuration space is given by $M \times N$, and $\Gamma(M \times N)$ is space of $N$-valued functions of spacetime. These functions are generally not continuous as in the case of 2 -valued configuration space.

2) $\boldsymbol{R}$-valued configuration space

Dividable and continuous physical values are represented by set of real numbers : $\boldsymbol{R}$. If physical quantity has no minimum element, it is dividable and has structure isomorphic to set of rational numbers : $\boldsymbol{P} . \boldsymbol{R}$ is topological completion of $\boldsymbol{P}$ and therefore continuous. Examples of physical quantities represented by $\boldsymbol{R}$ are density, temperature, pressure, and other classical ones.

Concept of real numbers is fundamental in physics because all measurable physical quantities are represented by discrete or continuous spectra on the field of real numbers. Thus physical status in classical physics is completely described in n-dimensional space of real numbers : $\boldsymbol{R}^{n}$, and the bundle structure is given by $M \times$ $\boldsymbol{R}^{n}$. Cross sections $\Gamma\left(M \times \boldsymbol{R}^{n}\right)$ corresponding to set of physical statuses is collection of $\boldsymbol{R}^{n}$-vector valued functions over spacetime. $\boldsymbol{M} \times \boldsymbol{R}^{n}$ structure is an appropriate configuration space to describe classical physical phenomena.

3) C-valued configuration space

Set of complex numbers: $\boldsymbol{C}$ is obtained as an algebraic enclosure of $\boldsymbol{R}$. Because complex numbers cannot be oberved directly as physical quantities, they must be accompanied with some algebraic operation procedures to be measured. In other words, a physical observation is decomposed into the status and the mesurement procedure. Therefore bundle structure $\boldsymbol{C}^{n}$ is an appropriate frame to represent non-classical physical configuration space.

$\Gamma\left(M \times C^{n}\right)$ is collection of $C^{n}$-vector valued functions over spacetime : i.e. wave functions. For example, $\gamma \in \Gamma\left(M \times C^{n}\right)$ is represented by wave function $f$ : $M \rightarrow C^{n}$, and we can obtain an observable physical quantity by algebraic operation $f^{*}(x) f(x)$. We may interpret the quantity as a squared wave amplitude of electromagnetic field or a probability density of quantum state vector. Therefore physical 
interpretations are necessary in order to apply $\Gamma\left(M \times C^{n}\right)$ and the algebraic operations to concrete physical theories.

4) G-valued configuration space

More generalized configuration space which appeares in physics is given by $M \times$ $G$, where $G$ is a topological group. Of course $R^{n}$ and $C^{n}$ are examples of topological groups with additive structure, and $G$ is locally isomorphic to $\boldsymbol{R}^{n}$ or $\boldsymbol{C}^{n}$. Therefore $M \times G$ is a natural extension of $M \times R^{n}$ or $M \times C^{n}$.

An example of $M \times G$ appeares as generalized Yang-Mills field in the theory of elementary particle physics. And $\Gamma(M \times G)$ is set of the group valued functions to describe the Yang-Mills field.

Logical evolution sequence of the generalized configuration space is depicted as follows.

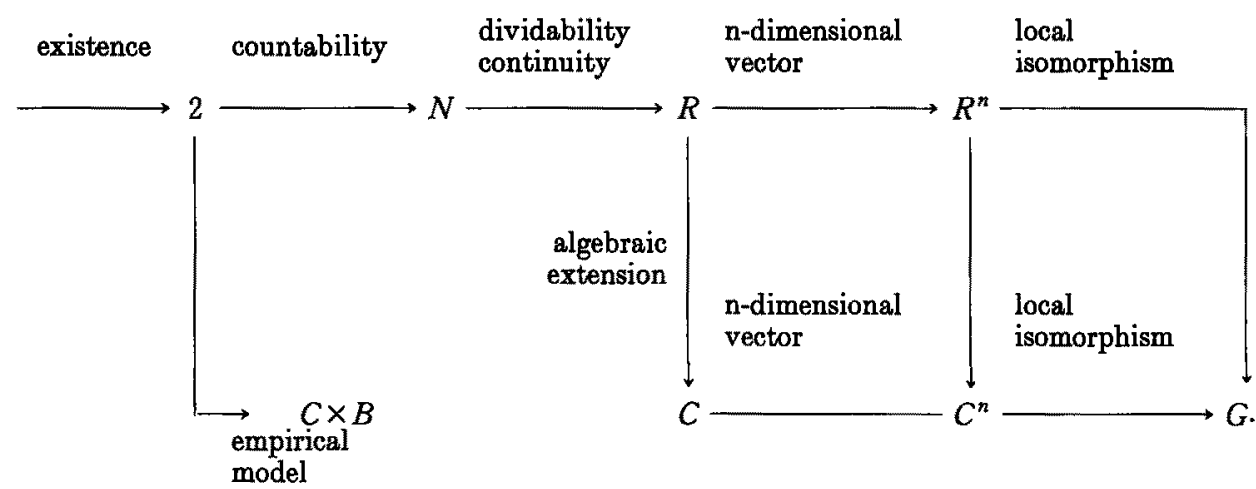

There is another possibility that fibre is operator space as in quantum field theory. The corresponding generarized configuration space is described as the following diagram ;

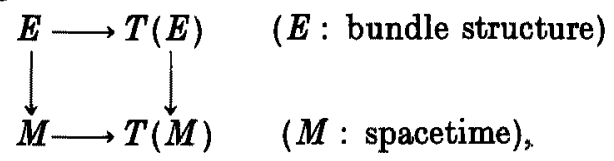

where $T$ means tensor bundle. The detailed discussion will appear in another paper in preparation.

\subsection{Locality and Globality}

Our knowledges about world are limited not only in the region size of spacetime but also in the amount of physical quantities. On our local frame of reference, a representation of configuration space is given by $r: E \mid U_{\alpha} \rightarrow U_{\alpha} \times F_{\alpha}$, where $E \mid U_{\alpha}$ is restriction of bundle structure $E$ to the spacetime region $U_{a}$ and $r$ is local homomorphism. Suppose that another representation of $E$ is given by $r^{\prime}: E \mid U_{a}^{\prime} \rightarrow$ 
$U_{a}^{\prime} \times F_{\alpha}^{\prime}$. If $U_{a} \cap U_{a}^{\prime} \neq 0$ and $r \sim r^{\prime}$ (i.e. $F_{\alpha} \sim F_{a}^{\prime}$ ), local configuration space can be extended to $U_{\alpha} \cup U_{\alpha}^{\prime}$. In the patch-work extension procedure, we can obtain our whole configuration space of physical world.

But the recursive procedure does not practically terminate because spacetime is practically infinite. Therefore we must posit that $\left\{F_{a}\right\} / \sim$ (equivalence class of local fibres) is represented by universal fibre $\boldsymbol{F}_{\boldsymbol{a}}$. Thus generalized configuration space will finally have a paradigmatic structure $M \times F$. Conceptual evolutions of physical theories correspond not only to the logical extensions of spacetime but also to those of fibre space.

\subsection{Equation of Motion}

One of the pragmatic objectives of physical sciences is to predict spatial and chronological variations of physical statuses. For given local configuration space $U_{\alpha} \times F_{\alpha}$ and the local cross section $\Gamma\left(U_{a} \times F_{a}\right)$ how can we obtain whole $E$ and $\Gamma(E)$ such that $E \mid U_{\alpha}=U_{a} \times F_{\alpha}$ and $\Gamma\left(E \mid U_{\alpha}\right)=\Gamma\left(U_{\alpha} \times F_{\alpha}\right)$ ? For another $U_{\beta} \subset M$, does there exist a structure preserving map (or isomprphism) such that $g_{\alpha \beta}: U_{\alpha} \rightarrow U_{\beta}$ and $g_{a \beta}^{*}: F_{\alpha} \rightarrow F_{\beta}$ ? If a pair of basic local configuration space $U_{0} \times F_{0}$ and a collection of the structure preserving maps $\left(g_{0 a}, g_{0 a}^{*}\right)$ exists, it completely generates whole $\bigcup_{\alpha} U_{\alpha} \times \mathrm{F}_{\alpha}$. If $\bigcup_{\alpha} U_{\alpha}$ coincides with $M$, the equation of motion is completely solved.

Usual form of the equation of motion is the special case that $U_{0}$ coincides a world point $x_{0}$. In that case, $g\left(x_{0}, x\right)$ is an element of the topological group which connects all spacetime point $x$ to $x_{0}$, and the equation of motion is presented as follows :

$$
g^{*} f(x)=U(g ; x) f\left(g^{-1} x\right) \quad(\text { for } f \in \Gamma(E)),
$$

where $U(g ; x)$ is representation of $g \in G$ on $\Gamma(E)$. This equation enables us to obtain necessary physical informations over the whole spacetime from the local fraction of the physical object.

\section{Conclusions}

We have presented that bundle structure is an appropriate logical scheme to reconstruct physical concepts in it. In the scheme, logical evolutions of physical concepts correspond to horizontal and vertical extensions of the bundle structure.

Horizontal extension corresponds to the conceptual evolution of spacetime. The evolution sequence of Galileian, Newtonian, Minkowski's and Einstein's concepts of spacetime is represented by the categorical sequence of the manifolds as $\left(E^{2} \times R\right) \times R, E^{3} \times R, M^{4}$, and $M$ respectively. 
Vertical extension corresponds to the conceptual evolution of quantitas materiae. Geometric and algebraic structure of fibre space represents measurement and observation procedure of the objective physical quantities. The evolution sequence of classical mechanics, quantum mechanics, and elementary particle physics is characterized by the corresponding fibre spaces as $R^{n}, C^{n}$, and $G$ respectively.

In the scheme of bundle structure, all physical statuses are described as local or global cross sections (i.e. $\Gamma(E \mid U)$ or $\Gamma(E)$ respectively) and all physical attributes and quantities are derived from the geometric and algebraic structures of the cross sections. Therefore to solve the equation of motion reduces to obtain the maximum extension of the given local cross sections. 